\title{
PPPL-2 128
}

\section{AR- 0387.5 PPPL-2128}

$20 / 19$

$$
\text { ( OC2O-F,G }
$$

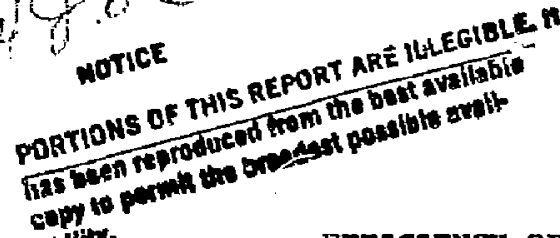

By

C.F.F. Karney and N.J. FAsch

AUGUST 1984

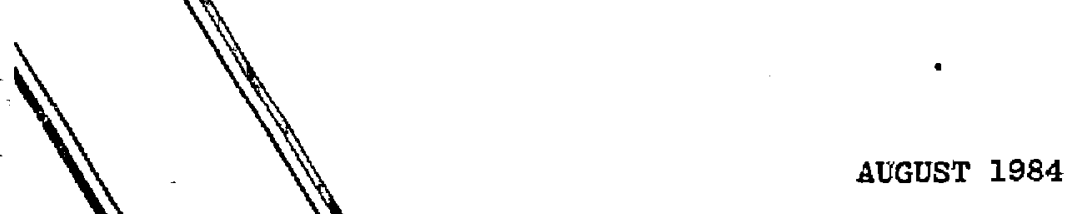

4

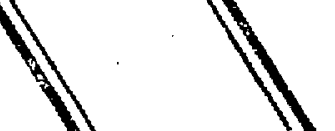

EFFICIENCY OF CURRENT DRIVE BY FAST TAVES

By

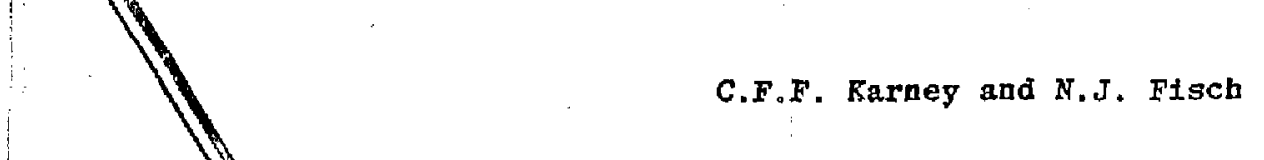

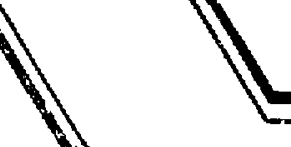

Lat

PLASMA

PHYSICS

LABORATORY

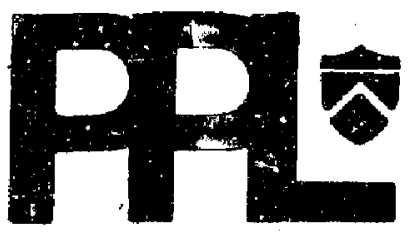

PRINCETON UNIVERSITY

PRINCETON, NEW JERSEY

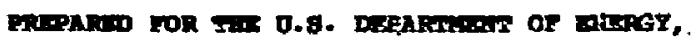

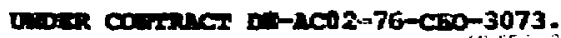


PPPE--212E

DEEA 017064

\title{
Efficiency of Current Drive by Fast Waves
}

\author{
Charles F. F. Karney and Nathaniel J. Fisch \\ Plasma Physics Lahoratory, Princeton University \\ Princeton, NJ 08544
}

The Rosenbluth form for the collision operator for a wearly relativistic plasma is derived. The formalism adopted by Antonsen and Chu can then be used to calculate the efficiency of current drive by fast waves in: a relativiatic plagma. Accurate numerical results and analytic aymptotic limits for the efficiencies are given.

\section{DISCLAIMER}

This report was prepared as an account of work 5ponsored by an agency of the United States Government. Ncithet the United Stutes Government nor any agency therenf. nor any of their employes, makes any warranty. express or implied, or assumes any Itgal liability or responsibility for the aceiracy, completeness, or usefulness of any information. apparalus, product, or process disclused, or represents that its use would not infringe privalely owned rights. Reference herein to any specific commescial prodtech, provess, or service by teade namc, trademark, manufucturer, or otherwise does not necessarily constitute or imply its endorsement, recommendation, or favoring by the United States Government or any agency thereof. The views and opinions of authors expressed herein do not necessarily statc or remecl those of the United States Government or any agency thereof. 


\section{INTRODUCTION}

Currents may be efficiently generated in a plasma by the injection of if waves whose phase velocities are several times the electron thermal speec! I The effeiency, defined as the ratio of current geaersted to pawer dissipated, is achieved in this ingtance becaure the rf-generated plateau decays at a rate given by the collision frequency for the fast electrons, which is relatively low. In the quest for higher efficiencies, current drive by waves which interac: with relativistic electrons has also been considered. ${ }^{2}$ Relativistic effects modify the scalin ., of the efficiency, placing an upper bound on the efficiency achievable by current drive by fagt, waves. In this paper, we do several things: we give a more complete anilyois of this problem based on a formalism adopted by Antonsen and Chu ${ }^{3}$ Specifically, we find that the effect of finite electron temperature leads to an enhancement of the efficiency. In order to calculate tbis effect, we first give expressions for the most important terms in the electron-electron collision integral in the relativistic limit. These expresaions are put in Rosenbluth form so as to be amenable to easy implementation on a computer. We imagine that the relativistic Rosenbluth potentials that we identify may be useful in other problems arising in very hot plamas.

In order to put the present work in perapective, let ua briefly review the chief tools used in the study of current drive. The early work used fairly crude analytical models. ${ }^{1,4}$ These modelo were suficient to obtain the scaling laws for the efficiency of cursent drive, but were unable to provide the coefficienta with any accuracy. Therefore, the analytical treat tent was supplemented by numerical solutions to the two-dimengional (in momentum space) Fokker-Planck equa-

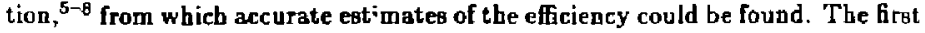
accurate analytical treatment of current drive was based on a Langevin formulation of the electron motion. ${ }^{2,8}$ This involved taking tbe electron temperature to be small, allowing energy acatteritg to be ignor-d. The moment bierarchy for the Langevin equations can then be closed, whict. allows an andytical solution to be obtained. This was followed by a more complete numsrical atudy of the Fokker-Planck equation for current drive in whici, the problem was reduced to the numerical solutio. of a one-dimensional integs o-differential equation with a source due to the $\mathrm{r}^{1}$. In this work toroidal effuts were also included. The results agreed with the Langevin analyois ${ }^{8}$ in the limit of large phase velocities (as they should) and gave more accurate numerical data for phase velocities comparable to or amaller than the the nal velocity. More recently, Antonsen and $\mathrm{Chu}^{3}$ and, independently, Taguchi, ${ }^{+*}$ using methods frst used in the study of beam-driven curreata, ${ }^{12,19}$ recognized that it is not necessary to solve the rf-driven Fokker-Planck equation in order to find the rf-induced current. Inatead, they showed that the Green'u function for the current is the Spitzer-Härm function 14 describing the perturbed electron distribution in the presence of an electric field. This reduces the problem to the determination of a single twodimensional function, from which the current generated by any torm of if drive 
can be calculated by a simple integration.

Up until now, the only reliable analytical results for current drive in a relativistic plasma are those obtained using the Langevin methodo by Ref. 2. As we will show, these are only exact for $T_{a}<m_{c} c^{2}$ and $p^{2}>m_{c} T_{c}$ (where $p$ is the momentum of the resonant electrons). A more complete analytical or numerical treatment along the lines of that achieved in the nonrelativistic case was bampered by the lack of a convenient form for the relativistic collision opetstor. This is remedied to some extent by the results of this paper where we calculate the colliaion integralo for the first Legendre harmonic oi the perturbed electron distribution neglecting electromagnetic effects on the binary interaction (in this approxiration the colligion integral reduces to the Landau form). Having done this, we are able to gereralize the trestment of Antonsen and Chu ${ }^{3}$ to the relativistic case. A number of useful results flow from this: we can numerically calculate to high precinion the current-drive efficiencieg in the relativistic tegime. We can perform an asymptotic andysis of the Spitzer-Häm problem to obtain analytic approximations to the efficiencies. In addition, we give higher-order asymptotic correctiond to the current-drive efficiencies in the nonrelativiatic limit. Throughout this paper, toroidal effects are entirely ignored. Although these effects are important in the study of current drive by low-phasevelocity wayes, they play little role in current drive by fast waves. Incorporation of these effects, bowever, proceed, in exact analogy with the treatment for the nonrelativistic case."

Relativistic effects on if curreni drive have aloo been considered by Hizsnidis and Bers. ${ }^{15}$ They attempt to determine the efficiency uing the moment equations given by Fisch. ${ }^{2}$ However, their subsequent analygis is flawed and their results for the current-drive efficiency are incorrect.

The plan of this paper is as followg: In Sec. II we show how the relativistic collision operator may be reduced to the Landou form. In this form, the colligion operator is costly to evaluate numerically. So, in Sec. III we convert the collision integrals to a Rosenbluth form, which may be evaluated very efficiently. The formulation of Antonsen and Chu is generalized to the relativistic case in Sec. IV. The numerical resulte for the efficiencies are given in Sec. $V$ and ibe asymptotic results in Sec. VI. Finally, in Sec. VII, we examine the agymptotir form of the efficiencies using the full relativistic collision operator.

\section{RELATIVISTIC COLLISION OPERATOR}

The collision operator for a relativiatic plasma is given by Beliaev and Budker. ${ }^{16}$ They give the collision operator as

$$
\left.\frac{\partial f_{a}(\mathbf{p})}{\partial t}\right|^{\text {coll }}=\sum_{b} C\left(f_{a}, f_{b}\right),
$$




$$
\begin{aligned}
C\left(f_{a}, f_{b}\right)= & \frac{q_{a}^{2} q_{b}^{2}}{8 \pi \epsilon_{0}^{2}} \log \Lambda^{a / b} \\
& \times \frac{\partial}{\partial \mathbf{p}} \cdot \int \mathbf{U} \cdot\left(f_{b}\left(\mathbf{p}^{\prime}\right) \frac{\partial f_{a}(\mathbf{p})}{\partial \mathbf{p}}-f_{a}(\mathbf{p}) \frac{\partial f_{b}\left(\mathbf{p}^{\prime}\right)}{\partial \mathbf{p}^{\prime}}\right) d^{\prime} \mathbf{p}^{\prime},
\end{aligned}
$$

where the lkernel $U$ is given by

$$
\begin{aligned}
& \mathrm{U}=\frac{\gamma_{a} \gamma_{b}^{\prime}\left(1-\beta_{a} \cdot \beta_{b}^{\prime}\right)^{2}}{c\left[\gamma_{a}^{2} \gamma_{b}^{\prime 2}\left(1-\beta_{a} \cdot \beta_{b}^{\prime}\right)^{2}-1\right]^{3 / 2}}\left\{\mid \gamma_{a}^{2} \gamma_{b}^{\prime 2}\left(1-\beta_{a} \cdot \beta_{b}^{\prime}\right)^{2}-1\right] 1 \\
&\left.-\gamma_{a}^{2} \beta_{a} \beta_{a}-\gamma_{b}^{\prime 2} \beta_{b}^{\prime} \beta_{b}^{\prime}+\gamma_{a}^{2} \gamma_{b}^{\prime 2}\left(1-\beta_{a} \cdot \beta_{b}^{\prime}\right)\left(\beta_{a} \beta_{b}^{\prime}+\beta_{b}^{\prime} \beta_{a}\right)\right\} .
\end{aligned}
$$

Here $a$ and $b$ are species labels, $q_{1}$ is the charg $=$ of species $\theta_{1} \log \Lambda^{a / b}$ is the Coulomb logarithm, $\epsilon_{0}$ is the dielectric constant, $P$ is the momentum, $v_{B}=$ $c \beta_{o}=\mathbf{p} / \mathbf{m}_{\mathrm{s}} \gamma_{\mathrm{s}}$ is the velocity of species $s_{\text {, and }} \gamma_{s}=\left(1+p^{2} / \mathbf{m}_{\mathrm{a}}^{2} c^{2}\right)^{1 / 2}$. The distributions are normalized so that

$$
\int f_{s}(\mathbf{p}) d^{s} \mathbf{p}=n_{*}
$$

the number density. We are primarily interested in situations where fast electrong are : vlliding off a weskly relativistic background. In that case $\beta_{b}^{\prime}<1$, and we can approximate $U$ by ita noarelativiatic form

$$
\mathbf{u}=\frac{u^{3} \mathbf{I}-\mathbf{u u}}{\mathbf{u}^{\mathbf{s}}}, \quad \mathbf{u}=\mathbf{v}_{\mathbf{a}}-\mathbf{v}_{\mathrm{b}}^{\prime} .
$$

Since the original form for $\boldsymbol{U}$ was symmetric in the primed and unprimed variables, we could equally well have obtained Eg. (1c) under the assumption that $\beta_{a}<1$. The relative difference between Eqs. (1c) and (2) is $O\left(\beta_{b}^{\prime}\right)$. However, the error in the collisios operator $C\left(f_{a}, f_{b}\right)$ is amaller than this. This point is examined in more detail in Sec. VII. Equatione (1) are precisely the collision operator given by Landau. ${ }^{17}$ Indeed an examination of his derivation shows that the mechanics of the collisions are treated relativistically; the interaction, however, is calculated nonrelativistically assuming a Coulomb potential. Use of Landau colliaion operator implies a neglect of the relativistic (i.e., electromagnetic) effects on the binary interaction. What we bave shown bere is that: such an approximation is valid provided at least one of the colliding particles is nonrelativistic.

It is readily eatablished that Eqs. (1) conserve number, momentum, and euergy $\left(\varepsilon_{a}=m_{a} c^{2} \gamma_{a}\right)$, that an $H$-theorem applies, and that the equilibrium solution is a relativistic Maxwellian $f_{a}(\mathrm{p}) \propto \exp \left(-\varepsilon_{a}^{\prime} / T\right)$, where $\varepsilon_{a}^{!}=\left(\varepsilon_{\mathrm{a}}-\right.$ $\left.\mathrm{v}_{d} \cdot \mathrm{p}\right) / \sqrt{1-v_{d}^{2} / c^{3}}$ is the energy in a frame moving at $\mathrm{v}_{d}$, and $T$ and $\mathrm{v}_{d}$ are independen: of the species $s$.

Throughout the rest of tbis paper we will restrict our attention to an electronjon plasma. We assume the ions are stationary and infinitely may ive $\left(m_{i} \rightarrow \infty\right)$. 
Thiz allows us to express the electron-ion collision operator in $(p, \mu)$ epace (where $\mu=p_{\|} / p$ and $\|$ and $\perp$ are with respect to the magnetic field) as

$$
C\left(f, f_{i}\right)=\Gamma \frac{Z}{2 u p^{2}} \frac{\partial}{\partial \mu}\left(1-\mu^{2}\right) \frac{\partial}{\partial \mu} f(\mathbf{p}) .
$$

where

$$
\begin{aligned}
& \Gamma=\frac{n_{e} q_{q}^{q} \log A^{e / e}}{4 \pi \varepsilon_{g}^{2}}, \\
& Z=-\frac{q_{i} \log \Lambda^{\alpha / 4}}{q_{e} \log \Lambda^{e / 0}} \approx-\frac{q_{i}}{q_{a}},
\end{aligned}
$$

asd we have assumed peutrality $q_{0} n_{\phi}+q_{i} n_{i}=0$. In Eq. (3) and beacelortb we will omit the species labels from all electron aquantities.

\section{GENERALIZATION OF THE ROSENBLUTH PO- TENTIALS}

For computatioasal purposes, the Landau operstor is not the most convenient form for the collision operator. If the plasma is aximutbally symmetric, a twodimensional integration must be performed at each point in momentum space. If the number of grid points is $N \times N$, this requires $O\left(N^{4}\right)$ calculations. This requirement is dramatically reduced in the non relativistic case by expressing the colligion operator in terms of Rosenbluth potentials. ${ }^{18}$ Unfortunately, although the Landau operator can be used without change to describe the collisions in a relativistic Coulomb plasma, the Rosenbluth form no longer applies. (The derivation of the Rosenbluth form from the Landau form requires, for ingtance, that $(\partial / \partial \mathbf{p}) \cdot \mathbf{U}=-\left(\partial / \partial \mathbf{p}^{\prime}\right) \cdot \mathbf{U}$, a relation that only bolds nonrelativiatically. $)$

However, hecause the kernel of the collision integral Eq. (1c) has the same form as in the nonrelativistic cage, it is possible to borrow some of the techniquea of Ref. 18. We convert the $p^{\prime}$ integration in Eq. (1b) to $r^{\prime}$ apece, subatitute a particular Legendre component for $f^{\prime}\left(p^{\prime}\right)$, and manipulate the resulting integrals into the form

$$
\int\left|v-v^{\prime}\right| P_{k}\left(\mu^{\prime}\right) h\left(v^{\prime}\right) d^{3} v^{\prime}
$$

or

$$
\int\left|v-v^{\prime}\right|^{-1} P_{k}\left(\mu^{\prime}\right) h\left(v^{\prime}\right) d^{3} v^{\prime}
$$

which may be evaluated in the same way as Rosenbluth potentials ${ }^{18}\left(P_{k}\right.$ is a Legendre polynomial).

Here we give the resulting expressions for collisions off a stationary Maxwellian background, i.e., $C\left(f, f_{m}\right)$, and for colligions of a Maxwellian off the first iegendre component of a baciground, i.e., $C\left(f_{m}, \mu f_{1}\right)$. ln both cases only electron-electron collisions are considered. These terms sre all that are required 
for the solution of the Spitzer-Hirm: problem (giving the Greed's function for the $\mathrm{f}$ current drive) and they suffice for an accurate numerical solution of the two-dimengional Fokker-Planck equation as described in Sec. V.

Beginning with the case of colligions off a Maxwellian, let us start by assuming merely that the background is irotropic $f(\mathbf{p})=f_{0}(p)$. The three-dimengional integrals in Eq. (1b) then reduce to ont-dimensional integrals giving

$$
\begin{aligned}
C\left(f, f_{0}\right)=\frac{1}{p^{2}} \frac{\partial}{\partial p} p^{2}(A(p) & \left.\frac{\partial}{\partial p}+F(p)\right) f(p) \\
& +\frac{B(p)}{p^{2}} \frac{\partial}{\partial \mu}\left(1-\mu^{2}\right) \frac{\partial}{\partial \mu} f^{\prime}(p)
\end{aligned}
$$

where

$$
\begin{aligned}
& A(p)=\frac{4 \pi \Gamma}{3 n}\left[\int_{0}^{p} p^{\prime 2} f_{0}\left(p^{\prime}\right) \frac{v^{\prime 2}}{v^{3}} d p^{\prime}+\int_{p}^{\infty} p^{\prime 2} f_{0}\left(p^{\prime}\right) \frac{1}{v^{\prime}} d p^{\prime}\right], \\
& F(p)=\frac{4 \pi \Gamma}{3 n}\left[\int_{0}^{p} p^{\prime} f_{0}\left(p^{\prime}\right) \frac{3 v^{\prime}-v^{3} / c^{2}}{v^{2}} d p^{\prime}+\int_{p}^{\infty} p^{\prime} f_{0}\left(p^{\prime}\right) 2 v / c^{2} d p^{\prime}\right], \\
& B(p)=\frac{4 \pi \Gamma}{3 n}\left[\int_{0}^{p} p^{r 2} f_{0}\left(p^{\prime}\right) \frac{3 v^{2}-v^{\prime 2}}{2 v^{3}} d p^{\prime}+\int_{p}^{\infty} p^{\prime 2} f_{0}\left(p^{\prime}\right) \frac{1}{v^{\prime}} d p^{\prime}\right] .
\end{aligned}
$$

Specializing 'o the case $f_{0}=f_{\mathrm{m}}$ and using the relation $\partial f_{\mathrm{m}} / \partial p=-(0 / T) f_{\mathrm{m}}$, we find that

$$
F(p)=(v / T) A(p)
$$

and the steady-state solution to $C\left(f, f_{m}\right)=0$ is that $f$ is a relatiriatic Maxwelliag ${ }^{19}$ with temperature $T$

$$
f_{m}(p)=\frac{n}{4 \pi m^{2} c T \hbar_{2}\left(\theta^{-1}\right)} \exp (-\varepsilon / T),
$$

where

$$
\begin{aligned}
& \varepsilon=m c^{2} r, \\
& \theta=\frac{T}{m c^{2}}
\end{aligned}
$$

( $\theta=1$ correaponds to an electron temperature of $511 \mathrm{keV})$, and $K_{n}$ is the nth-order modified Bessel function of the second kind.

For later use we define bere a thermal momentum

$$
p_{i}=\sqrt{m+\bar{T}},
$$

a mean-squared velocity

$$
\begin{aligned}
v_{t}^{3} & =\frac{1}{3 n} \int v^{2} f_{m}(p) d^{3} \mathbf{p}=\frac{T}{m} V_{t}^{2}, \\
V_{t}^{2} & =1-\frac{5}{2} \theta+\frac{55}{8} \theta^{2}+O\left(\theta^{3}\right),
\end{aligned}
$$


a thermal ccllision frequency

$$
\nu_{t}=\frac{m \Gamma}{p_{t}^{9}}=\frac{n q^{4} m \log A}{4 \pi \epsilon_{0}^{2} p_{t}^{9}},
$$

and a collision frcquency normalized to the speed of light

$$
\mu_{c}=\frac{\Gamma}{m^{3} c^{3}}=\frac{n q^{4} \log \Lambda}{4 \pi \epsilon_{o}^{2} m^{2} c^{3}}
$$

Note that these frequencies differ by a factor of two from those used in earlier publications. $1,2,5,8, a, 8$ Specifically, we have $\nu_{t}=\nu_{0} / 2$ and $\nu_{e}=\nu / 2$. This meang that all our normalized efficiencies are a factor of two smaller than in these earlier papers. (We made this change hecause the normalized Fokker-Planck equation in the high-energy limit now has a aimpler form. This convention is alto used by other workers in this field.)

For $p>p_{t}$, the indefinite limitg in the integrala in Eq. (1) can be replaced by $\infty$, giving ${ }^{20}$

$$
\begin{aligned}
& A(p)=\Gamma \frac{v_{l}^{2}}{v^{s}} \\
& B(p)=\Gamma \frac{1}{2 v}\left(1-\frac{v_{t}^{d}}{v^{2}}\right) .
\end{aligned}
$$

Note that the frictionb! force $F(p)$ reachea a constant value as $p \rightarrow \infty$. This implies, for instance, that an electric field amaller than $\Gamma v_{l}^{2} / q T c^{2}$ cannot produce runaways. ${ }^{21}$ On the other hand, the pitch-angle scattering frequency $B(p) / p^{2}$ continues to decay as $p \rightarrow \infty$. As the energy of the electron increases, its effective mass increases. It is then more dificult to deflect the heavier particle. In this limit, pitch-angle acat tering is negligible compared with frictional slowing down. This is to be contrasted with the nonrelativistic case where the pitchangle scattering frequency and the frictional slowing-down rate decay as $1 / p^{2}$ and the two processes are of comparable importance.

The implication for current drive is that the efficiency of parallel waveinduced fuxes, say by lower-hybrid waves, approaches a conotant. This can be seen as followe: Nonrehativistically, the efficiency increases as $p^{2}$. Relativit tic electrons, however, slow down faster because they are heavier, and they also do not carry more current when pughed in the parallel direction. Each of these effects reduces the efficiency by $\gamma \sim p$; hence the approach to a constant.

The other term we shall need is $C\left(f_{m}, \mu f_{1}\right)$. This term is rather barder to compute. We define $f_{1}(p)=f_{m}(p) \chi_{1}(p)$ and write $C\left(f_{m}, \mu f_{m} \chi_{1}\right)=\mu f_{m} I\left(\chi_{1}\right)$. Again, we reduce (this time after much algebra) the integrals in Eq. (1b) to one-dimensional ones to give

$$
I\left(x_{2}\right)=\frac{4 \pi \Gamma}{n} x
$$




$$
\begin{aligned}
& \left\{\frac{m f_{m}(p) \chi_{1}(p)}{\gamma}\right. \\
& +\frac{1}{5} \int_{0}^{p} p^{\prime 2} f_{m}\left(p^{\prime}\right) X_{2}\left(p^{\prime}\right) \frac{m}{T}\left[\frac{\gamma}{p^{2}} \frac{v^{\prime}}{\gamma^{\prime 3}}\left(\frac{T}{m c^{2}}\left(4 \gamma^{\prime 2}+6\right)-\frac{1}{3}\left(4 \gamma^{\prime 3}-9 \gamma^{\prime}\right)\right)\right. \\
& \left.+\frac{\gamma^{2}}{p^{2}} \frac{v^{\prime}}{\gamma^{\prime 3}}\left(\frac{m v^{\prime 2}}{T} \gamma^{\prime 3}-\frac{1}{3}\left(4 \gamma^{\prime 2}+6\right)\right)\right] d p^{\prime} \\
& +\frac{1}{5} \int_{p}^{\infty} p^{\prime 2} f_{m}\left(p^{\prime}\right)_{X_{1}}\left(p^{\prime}\right) \frac{m}{T}\left[\frac{\gamma^{\prime}}{p^{\prime 2}} \frac{v}{\gamma^{3}}\left(\frac{T}{m c^{2}}\left(4 \gamma^{2}+6\right)-\frac{1}{3}\left(4 \gamma^{3}-9 \gamma\right)\right)\right. \\
& \left.\left.+\frac{\gamma^{\prime 2}}{p^{12}} \frac{v}{\gamma^{3}}\left(\frac{m v^{2}}{T} \gamma^{3}-\frac{1}{3}\left(4 \gamma^{2}+6\right)\right)\right] d p^{\prime}\right\}
\end{aligned}
$$

The term in square brackets in the last integral matches that in the first integral except for the interchange of the primed and unprimed variables. The simplification of Eq. (7) was achieved, in part, with the belp of the symbolic manipulation program, MACSYMA. ${ }^{22}$

Equations ( 4 ) and $(7)$ are now in a computationally conveniegl form. Their evaluation involves the determination of a number of indefinite integrals (the unprimed variables should be factored oul of the integrals for this step), and the multiplication of these integrals by various functions of $p$. If the distribution functions are known on a grid of $N$ points, then the computational cost is just $O(N)$. Furthermore, the calculation can be arranged so that nearly all the computations vectorize. ${ }^{23}$ The general solution of the linearized electroselectrod collision operator $C\left(f, f_{m}\right)+C\left(f_{m}, f\right)=0$ is

$$
f=(a+b \cdot p+c \varepsilon) f_{m} .
$$

where $a, \nu$, and $c$ are arbitrary constants. With $a=c=0$ and $b=\hat{p}_{\|}$, this provides a useful cbeck on Eqs. (4) and (7) and their computational realizations.

\section{FORMULATION}

We now turn to the calculation of the if efficiency. There are three steps involved: the apecilication of the of currept-drive problem, the identification of the Spitzer-Häm function as the Green's function for the rf-driven current; and the solution of the Spitzer-Härm problem.

We begin with the specification of the problem. This is just a standard application of the Chapman-Enskog procedure. ${ }^{24}$ The most important assumption is that the collisional time scale is mucb shorter than the transport time scale (the time scale for heating the plasma by the $\mathrm{r}$ ). This places some restrictions on the if drive. However, these are usually not severe ones in the case of fast-wave current drive because, even if ihe $r f$ is strong, there are few vesonant partic]es and, consequently, the heating rate is small. 
The effect of the $\mathrm{if}$ is to induce an electrou flux

$$
\mathbf{S}=-\mathbf{D} \cdot \frac{\partial f}{\partial \mathrm{p}}
$$

in momentum space, where $D$ is the quagilinear diffusion tensor. ${ }^{28}$ In the Chapman-Etrakos ordering this is taken to be of first order. The zeroth-order electron distribution is given by getting the collisinn term $C(f, \delta)+C\left(f, f_{i}\right)$ equal to zero. The general solution is a Maxwellian Eq. (5) with $n$ and $T$ arbitrary functions of time and position. For simplicity we iznore the apatial variations. Since the $r$ drive is particle conserving, we may take $n$ to be a constant. A drifting Maxwellian does not solve the zeroth-order system since the ions are take: to be stationary.

The first-order equation is given by subatituting $f=f_{m}(1+\psi)$ with $\psi$ ordered small to give

$$
C\left(\delta_{m} \psi\right)=\frac{\partial}{\partial \mathbf{p}} \cdot \mathbf{S}+\frac{(\varepsilon-\langle\varepsilon\rangle)}{T} f_{m} \frac{d}{d t} \log T,
$$

where

$$
C(f)=C\left(f, f_{m}\right)+C\left(f_{m}, f\right)+C\left(f, f_{i}\right)
$$

is the linearized collision operator, and $\langle\varepsilon\rangle$ is the mean energy per particle ${ }^{19}$

$$
\begin{aligned}
\langle\varepsilon\rangle & =\frac{1}{n} \int \varepsilon f_{m}(p) d^{2} \mathrm{p} \\
& =m c^{2}\left(\frac{K_{1}\left(\theta^{-1}\right)}{K_{2}\left(\theta^{-1}\right)}+3 \theta\right) .
\end{aligned}
$$

The last tcrm in Eq. (9) represents the heating of the Maxwellian. The equation for the time evolution of $T$ is given by the solubility condition for Eq. (9), which is obtained by taking its energy moment. Since the lineariged collision operator is energy cocserving (recall that we take the limit $m_{i} \rightarrow \infty$, so that there is no energy exchange between electron and iona), this gives

$$
n \frac{d\langle E\rangle}{d t}=P
$$

where $P$ is the power disgipated per unit volume by the of

$$
P=\int \mathbf{S} \cdot \mathbf{v} \mathbf{d}^{\mathbf{s}} \mathbf{p} \text {. }
$$

(There is another solubility condition given by the density moment of Eq. (9). This is automatically satisfied by taking $d n / d t=0$.] The solution to Eq. (g) is made unique by denanding that $f_{m} \psi$ have zero dengity and evergy. 
In the nonrelativistic linit, Eq. (9) is the equation solved numerically by Cordey et al. ${ }^{10}$ However, since we are ugually interested primarily in the currept density generated by the of

$$
J=q \int v_{\|} f_{m} \psi d^{*} p,
$$

and the efficiency of current generation detined by the ratio $J / P$, we tugually do noc need to know she fall solution for $\psi$.

The method for determining tine current without golving for $\psi$ was given by Hirshman ${ }^{i 2}$ and Tagichi ${ }^{13}$ for neytral-beam-driven currents and was introduced into the study of rf-driven currents by Antonsen and $\mathrm{Chu}^{3}$ and Taguchi. ${ }^{11}$ The key is to define an "soljoint" problem

$$
C\left(f_{m} x\right)=-q v_{\|} f_{m} .
$$

Again $f_{m x}$ is required to have zero dengity and energy. This is the Spitzer-Härm problem for the perturbed electron distribution function due to an electric bild $\mathbb{E}=T \hat{\mathbf{p}}_{\mid i}$. Using the self-adjoint property of the linearized collision operator $\int \psi C\left(f_{m} \chi\right) d^{3} \mathbf{p}=\int x C\left(f_{m} \psi\right) d^{2} \mathbf{p}$, it is readily found that

$$
J=\int \mathrm{s} \cdot \frac{\partial}{\partial \mathrm{p}} \times(\mathrm{p}) d^{\mathbf{s}} \mathrm{p} .
$$

In this equation $x$ plays the role of a Green's function for the rf-driven current. The ratio of Eqa. (14) and (11) gives the efficiency

$$
\frac{J}{P}=\frac{\int \mathrm{s} \cdot \frac{\partial}{\partial \mathrm{p}} \times(\mathbf{p}) d^{3} \mathrm{p}}{\int \mathrm{s} \cdot \mathbf{v} d^{2} \mathbf{p}}
$$

An important speciai case is when the rf excitation is localized. 'Then it is only necessary to know the position and direction of the excitation to determine the efficiency

$$
\frac{J}{\vec{P}}=-\frac{\dot{\mathbf{s}} \cdot \frac{\partial}{\partial \mathbf{p}} x(\mathbf{p})}{\dot{\mathbf{S}} \cdot \mathbf{v}},
$$

witere all quantities are now evaluated at the position of the excitation. If we compare this method with the Langevin method of Fisch, ${ }^{3}$ we see that $\chi$ is the mean-integrated current due to a group of electrons released at $\mathrm{p}$ at $t=0$

$$
\chi(\mathbf{p})=q \int_{0}^{\infty}\left\langle v_{\|}\right\rangle d t .
$$

The power sf thege results is that the calculation of $J / P$ does not require a solution of Eq. (9) for the rf distribution $\psi$. On the other hand, Eq. (13) must be 
solved for the Spitzer-Härm function $x$. Thes reduces to the solution of a onedimensional integro-differential equation, which may be accurately computed. Furthermore, in the nonrelativistic limit, it has been tabulated. ${ }^{14}$ This method also substantially reduces the parameter space to be investigated numerically. The solution of Eq. (13) depende on two parameters only, $Z$ and $\theta$. In contrast, the solution of Eq. (9) depends on various parameters specifying the nature of the $f$ excitation (for instance, the direction of $\mathbf{S}$, the minimun and maximum phase velocities, etc.) as well as $Z$ and $\mathbf{\theta}$.

In order to determine the rf current-drive effejency ubing Eqs. (15) or (16), we must solve the Sp-Lzer-Härm problem, Eq. (13). The solution $x$ consists of only the first Legendre barmonic, so we substitute $\chi(p)=\mu \chi_{1}(p)$ into Eq. (13) giving

$$
\frac{1}{p^{2}} \frac{\partial}{\partial p} p^{2} A(p) \frac{\partial \chi_{1}}{\partial p}-\frac{v A(p)}{T} \frac{\partial \chi_{1}}{\partial p}-\frac{2 B(p)+\Gamma Z / v}{p^{2}} \chi_{1}+I\left(\chi_{1}\right)+q v=0
$$

where $A(p)$ and $B(p)$ are given by Eq. (q), the electron-ion tern is given by Fq. (3), and $I\left(x_{1}\right)$ is given by Eq. (7). The foct that the golution of Eq. (13) consists of only a singie Lregendre component constilutes an additional advantage to this method of determining current-drive efficiencies. The solution of the full If problein given in Eq. (9) consiate, in reneral, of many Legendre componests. Often eome truncation is performed in computing these nulierically.

Equaiion (17) may be solved by approximate analytic methods either by expressing $x$ a a sum of Sonine polynomialo 24,28 or by formulating the equation as a rariational problem. ${ }^{12}$ These methods have the disadvantage that they generally fail to reproduce the sorrect asymptotic (large p) form for $x$. This failing does not affect the calculation of the electrical conductivity signilicantly since in that case $X$ is integrated with a weighting factor proportional to $f_{m}$. However, it rules oui auch methods for the study of of current drive, since the eficiency may depend on the local value of $x$.

This leaves us either with asymptotic methods, which we apply in Sec. VI, or with numerical methods. Numerical solutions to $\mathrm{Eg}$. (17) bave been given in the nonrelativistic cage in Refa. 14 and 27 . Here we use a simpler method that avoids most of the problems with the application of boundary conditions. We cast Eq. (17) a one-dimensional difusion equation by eetting the lefthand side to $\partial_{\chi_{1}} / \partial i$ and solve this diffusion equation until a steady state is reached. (The initial conditions may be chosen arbitrarily.) The integration is carried out in the domain $0<p<p_{\text {mex }}$ and the boundary conditiong $\chi(0)=0$ and $\chi^{\prime \prime}\left(p_{\text {mex }}\right)=0$ are imposed. The diffusion equation describes the physical problem of the evolution of the perturbed electron distribution in the presence of an electric field and is therefore guaranteed to give the correct solution of Eq. (13) without having to worry about spuriouc solutions that diverge at $p=0$ or $p=\infty$. Since this is a one-dimezuional difusion equation, it may be solved by treating the differential operator fully impiicitly (the time step may be taken to be large). The integral operator $I\left(x_{1}\right)$ in treated explicitly and is recomputed 
after every time step. In the calculations shown here, the momentum step size was taken to be $p_{1} / 50$, the time step was taken to be $1000 / \nu_{t}$, and the process converged (i.e., the relative change in $\chi_{i}$ per step was less than 1 part in $10^{10}$ ) after about 50 steps.

In the following sections we will also need the function $G(p)=x_{1}(p) / p$ so that $x(p)=p_{\|} G(p)$. In terns of $G$, the gradient of $x$ is

$$
\frac{\partial}{\partial \mathbf{p}} \chi(\mathbf{p})=G(p) \hat{\mathbf{p}}_{\|}+\mathbf{p}_{i \mid} G_{p}(\mathbf{p}) \hat{\mathbf{p}}
$$

where $G_{p}(p)=d G(p) / d p$.

\section{NUMERICAL RESULTS}

The solution for $\chi$ is given as a contour plot in Fig. 1 for $Z=1$ and $\theta=0$ and 0.01 . From these and a knowledge of $\mathbf{S}$, the direction of the it-induced curreat can be determined. In the nor relativitic case, Fig. 1 (a), $x$ rises ever inore ateeply $a^{f} F$ is increased, giving the tavorable $p^{2}$ scaling for the curresthdrive efficien':y. ${ }^{*}$ On the other hand, in a hot plasma, Fig. 1(b), the vlope reaches a csustant (the contour levels are equally spaced), Jeading to a limit in the efficiency of the current drive. ${ }^{2}$

Figure 1 also shows that the contours become vertical for $p_{\|}$small. This indicates that pushing electrons with small $p_{\|}$in the perpendicular djrection (as with cyclotron-demped waves) is not effective in generiaing current. Puahing electrone in the parallel direction is effective, especially for small $p_{\|}$, since the denominacor in Eq. (16) can be small. In general, when the contours of constant anergy ( $p=$ corstaut) cross contoura of constant $\chi$, the efficiency can be very large.

Turning now to the numerical resulte for the efticiency, we uegin with the cuse of a localized ppectrum, Eq. (16). Although this situation may not be realized in practice, it is important becauge it can help us to determine the best currentdrive schemes by showing where in velocity space to induce the llux. There are two major classes of fast wave that have been considered for current drive, namely Landau-damped waves (e.g., lower-hybrid waver) for which $\hat{\mathbf{S}}=\hat{\mathbf{p}}_{\|}$and cyclotron-damped waves for which $\mathbf{S}=\hat{\mathbf{p}}_{\perp}$. Taking the limit $p_{\perp} \rightarrow 0$, we have

$$
\begin{aligned}
& \frac{J}{p}=\frac{G(p)+p G_{p}(p)}{v} \\
& \frac{J}{P}=\frac{p G_{p}(p)}{v}
\end{aligned}
$$

for Landau-damped and cyclotron-damped waves, respectjvely. The efficiencies are plotted in Fig. 2 for $Z=1$ and $\theta=0,0.02,0.05,0.1$, and 0.2 (these correspond to $T=0,10,26,51$, and $102 \mathrm{keV}$ ). The curves for $\theta=0$ in the two cases are given analytically from Eq. (24); they agree with the regults of 
Ref. 2. This confirns the earlier analysis and shows that it is exact in the limit of $T \ll m c^{2}$ and $p^{2}>m T$.

We next consider current drive by a narrow speciruge of iandau-damped waves. In this case, all particles satisfying the Landau resonance cupdition $w-k_{\|} v_{\|}=0$ interact with the wave, and the quasilinear diffusion tensor is

$$
\begin{aligned}
\mathbf{D} & \propto \delta\left(\omega-k_{\|} v_{\|}\right) \hat{\mathbf{p}}_{\|} \hat{\mathbf{p}}_{\|} \\
& \left.\propto \gamma \delta\left(p_{\|}-m\right)_{p} \gamma\right) \hat{\mathbf{p}}_{\|} \hat{\mathbf{p}}_{\|},
\end{aligned}
$$

where $v_{\mathrm{p}}=\omega / k_{\mid}$is the parallel wave phase velocity. Asguming that the electron distribution is weakly perturbed, we can take $f=f_{m}$ in Eq. (8) to give

$$
\mathbf{S} \propto \gamma v_{\|} f_{m} \delta\left(p_{\|}-m v_{p} \gamma\right) \hat{p}_{\|} .
$$

When we substitute tbis expression into Eq. (15), we obcain

$$
\frac{J}{P}=\frac{1}{v_{p}} \frac{\int_{p a}^{\infty}\left(G(p)+\frac{\left(m \gamma v_{p}\right)^{2}}{p} G_{p}(p)\right) \gamma f_{m}(p) p d p}{\int_{p_{0}}^{\infty} \gamma f_{m}(p) p d p},
$$

where $p_{0}=m v_{s} /\left(1-v_{p}^{2} / c^{2}\right)^{1 / 2}$ is the minimum resonant momeatum. This efficiency is plotted in Fig. 3. In the limit $v_{p} \rightarrow 0$, the efficiency be zomes large. This demonatrates that current may be efficiently driven by low phase velocity waves as vas propased by Wort. ${ }^{4}$

A vimiler analysig can he performed for a nartow spectrum of cyclotrondamped waves. The aituation is more complicated here because the electron cyclotron frequency depends relativistically on the momentum ${ }^{38}$ and recause relativistic effects distort the diffusion paths. ${ }^{8}$ In addition, the yariation of the diffusion cuefficient with $p_{\perp}$ depends on the harmonic number. This means that the efficiency depends on three wave parametera $\omega / k_{\|}, \Omega / k_{\|}$( $\Omega$ is the rest-mass cyclotron frequency), and the harmonic number. We therefore will only treat this cose in the gonrelativistic limit.

In the non relativistic limit $(\Theta \rightarrow 0, p / m e \rightarrow 0)$, the efficiencieg for both kinda of wave have been calculated by Cordey et al. ${ }^{10}$ and Taguchi. ${ }^{11}$ They' considered a narrow spectrum of Landau-damped waves for which the efficiency is given by the nonrelativintic limit of Eq. (19) and a narrow spectrum of cyclotrondamped waves for which the diffusion coefficient is

$$
\mathrm{D} \propto v_{\perp}^{2(l-1)} \delta\left(v_{\|}-v_{p}\right) \hat{\mathbf{p}}_{\perp} \hat{\mathbf{p}}_{\perp}
$$

where $l$ is the harmonic number and $v_{\mathrm{p}}=(\omega-l \Omega) / k_{\|}$. Assuming that $f=f_{m}$ in Eq. (8), the effectency for cyclotron-damped waves is

$$
\frac{J}{P}=m^{2} v_{p} \frac{\int_{p_{0}}^{\infty}\left(p^{2}-p_{0}^{2}\right)^{l} f_{m}(p) G_{p}(p) d p}{\int_{p_{0}}^{\infty}\left(p^{9}-p_{0}^{2}\right)^{l} f_{m}(p) p d p},
$$


where $p_{0}=\boldsymbol{n}_{\boldsymbol{n}} \mathrm{v}_{\boldsymbol{p}}$. (Here we cansider only the fundamental cyclotron resonance $t=1$ ) In Fig. 4, we plot these efficiencies normalized to the thermal quantitieg together with the asymptotic results, Eqs. (31) and (32a). For $m v_{p} \geqslant p_{t_{1}}$ the efficiencies scale as $v_{p}^{2}$ as predicted by Fisch and Boozer. 9 The $1 / u_{p}$ scaling seen in the Landau-damping case for $m v_{p}<p_{l}$ is obtained by taking the limit $v_{p} \rightarrow 0$ in Eg. (19) to give

$$
\frac{J}{P}=\frac{1}{v_{p}} \frac{\int D\left(p_{\perp}\right) f_{m}\left(p_{\perp}\right) G\left(p_{\perp}\right) p_{\perp} d p_{\perp}}{\int D\left(p_{\perp}\right) f_{m}\left(p_{\perp}\right) p_{\perp} d p_{\perp}}
$$

Hert we have included an arbitrary dependence of $D$ on $p_{\perp}$. In Ref. 6, three differeat types of low-phase-velocity current drive were identified, namely by Landau damping, transit-time magnetic pumping, and Allvén waves. These methods differ in the forms for $D\left(p_{\perp}\right)$

$$
D\left(p_{\perp}\right)= \begin{cases}1 & \text { (Landau damping), } \\ \left(p_{\perp} / p_{t}\right)^{4} & \text { (trangit-time magaetic pumping), } \\ \left\{2-\left(p_{\perp} / p_{t}\right)^{2}\right]^{2} & \text { (Alfvén waves). }\end{cases}
$$

The case plothed in Fig. 4 is the first one (Landau damping). Evaluating the integrals in these caseg gives

$$
\frac{J}{P}=\left\{\begin{array}{c}
C_{L} \\
C_{M} \\
C_{A}
\end{array}\right\} \frac{q}{m v_{p} \nu_{t}}
$$

where the coefficients $\sigma$ are given in Table $I$. The coefficients for $Z=1$ ghould be compared with the (less exact) results of Ref. $\theta$ abtained by a numerical ealution of the two-dimensional Fokker-Planck equation where the conatants of proportionality are given as $4,6.5$, and 6.5 , respectively. The coefficient $C_{L}$ tas been determined anolytically by Cordey $e t a d^{10}$ to be

$$
C_{L}=\frac{3 \sqrt{2 \pi}}{2 Z}
$$

The dependence on $Z$ ixdicates that the current is unaffected by electronelectron colligions. This result may be derived by taking the momentum moment of Eq. (13). The electron-electron collision term then drops out (from morrentum conservation) and the electron-ion term is proportional to the numerator in Eq. (21).

The last numerical example is one in which we relax the condition that $f=f_{m}$ in Eq. (8). This allowg us to find the flux $\mathbf{S}$ that develops in the prescnce of high if power. In order to determine $S$, we numerically solve tíe two-dimensional Folker-Planck equation

$$
\frac{\partial f}{\partial t}=C_{\text {num }}(f)+\frac{\partial}{\xi_{p}} \cdot D \cdot \frac{\partial f}{\partial \mathbf{p}},
$$


until a steady state is reached. The numerical collision operator is defined as

$$
C_{\text {num }}(f)=C\left(f, f_{m}\right)+C\left(f_{m}, \mu f_{1}\right)+C\left(f, f_{i}\right) \text {, }
$$

where $\mu f_{2}$ is the fisst Legendre barmonic of $f$. The electron-jon term $C\left(f_{t} f_{i}\right)$ is calculated using Eq. (3).

In order to justify our handling of the electron-electron collisions, let LG consider the linearized electron-electron operator $C\left(f, f_{m}\right)+C\left(f_{m}, f\right)$. The first term describes the relaxation of the tail particles on the bulk and the secund deacribes the concomitant heating of the bulk. The linearization is justified even with atrong $r$, as long as $f(p) \approx f_{m}(p)$ for $\varepsilon \sim T$. The linearized electronelectron operator conserves energy, and if this were used in Eq. (22), there would be notbing to balance the power input by the $\mathrm{rf}$ (there is no iranefer of energy to the jone in the limit $m_{i} \rightarrow \infty$ ), and so a steady-state solution to Eq. (22) would not be possible. In Eq. (9), this is handled by allowing the temperature of the Maxwellian to increase slowly with time. is the numericel code, we adopt a di Terent approach, namely to modify the collision operator so that energy is lost in an innocuous way. The term responsible for the bulk beating is the second terin $C\left(f_{m}, f\right)$. Let ut write $f$ in this term as a Legendre harmonic expansion

$$
f(\mathbf{p})=\sum_{k=0}^{\infty} P_{k}(\mu) f_{k}(p)
$$

Of the terms in this series, oply one, the $k=0$ term, contributes to the bulk heatiog. (The energy moments of the other terms vasish.) Thus in order to lose antrgy we drop the term $C\left(f_{m}, f_{0}\right)$. Of the remaining terms in the series, only the first, the $m=1$ term, is of importance-it is responsible for ensuring conservation of momentum. Thus we retain only this term and approxinate $C\left(f_{m}, f\right)$ by $C\left(f_{m}, \mu f_{i}\right)$ to give the collision operator $C_{\text {vum }}$.

The collision operator $C_{\text {num }}$ has the following properties: energy is not conserved (thus allowing a ateady at ate to be reached); momentum is conserved; and quantities euch as the Spitzer-Härm conductivity, which are given solely in terms of the fingt Legendre barmonic, are correctly given. To justify the way in which energy conservation is handled, we may check that the regulta are insensitive to the details of how this is done. One such check is given below where we compare the efficin ncy given by the numerical solution of Eq. (22), it which energy is lost, and that given by $\mathrm{Eq}$. (15), where energy is couserved.

We agsume that the $f$ diffusion term in Eq. (22) is caused by high-power lower-hybrid waves whose phase velocities lie between $v_{1}$ and $v_{2}$. Thus we take

$$
\mathbf{D}= \begin{cases}D(\mathbf{p}) \hat{\mathbf{p}}_{\|} \hat{\mathbf{P}}_{\|}, & \text {for } v_{1}<p_{\|} /(m \gamma)<u_{2}, \\ 0, & \text { otherwise }\end{cases}
$$

where $D(p)$ is chasen to be large enough to plateau $f$. [Here we choose $D(p)=$ $10 \nu_{t} p_{t}^{2} /\left(1+p / p_{t}\right)$.] 
Figure 5 ghows the eteady-state solution of $\mathrm{Eq}$. (22) for $Z=1, \theta=0.01$ $(T \approx 5 \mathrm{keV}), v_{1}=0.4 c=4 \mathrm{p}_{2} / \mathrm{m}$, and $v_{2}=0.7 c=7 p_{t} / \mathrm{m}$ (the parallel refractive index satisfieg $\left.1.43<n_{n}<2.5\right)$. Uning the numerical solution for $f(p)$ and $S(p)$, and the definitions (11) and (12), we obtain $J=3.74 \times 10^{-4} q$ ne, $P=$ $1.28 \times 10^{-3} \mathrm{mnc}^{7} \nu_{c}$, and $J / P=0.298 \mathrm{~g} / \mathrm{me}_{\mathrm{c}}$.

This is to be compared with the reault given by Eq. (14) with the numerically determined flux $S(p)$, eimely $J=3.77 \times 10^{-4} \mathrm{pnc}$ and $J / P=0.296 \mathrm{q} / \mathrm{mcts}$. (The figure for $P$ remains unchanged since this depends on $S$ alone.) These two sets of figures are within $1 \%$ of each other. The excellent agreement illisstrates two points: the approxinations made in the numerical collision operatior, namely, the teglect of the heating term $C\left(f_{m}, f_{0}\right)$, bas little effect on the resulte for the current-drive efficiencies (discretization effects are probably a greater source of error in these resulta); and the analytic result Fq. (15) can be used to obtain reliable figures for the efficiency for canes of strong $i f$. What is needed in the latter instance is an eatimate for the of fivx $\mathbf{S}$. This roay be found from a nutnerical solution of a two-dimensional Fokker-Planck equation (as bere) or from an approximate analytical solution. Some saving may be possible using this method in conjunction with a vmerical code: since $\$$ reaches a steady state sooner than $f_{1}$ it may not be necessary to run the code go long in order to obtain a reasonably accurate egtimate for the efficiency.

\section{ASYMPTOTIC ANALYSIS}

We have reen that the efficiency of current drive may be expressed in ieras of the solution of the Spitzer-Harm prublem, $\mathrm{Eq}_{\text {. }}$ (17). This equation may be approximately solved in the limit $p>p_{i}$. We will begin with the relativistic case and later treat the nonrelativistic limit. We start by witing down the normalized form of Eq. (17) in the limjt $p>p_{l}$. We chose normalizations based upon $q_{1} m_{1} c_{s}$ and $\nu_{\varepsilon}$. Thus momenta are normajized to $m c, x_{1}$ to $q c / \nu_{e}, J / P$ to $q /$ mec $\nu_{c}$ etc. We use the same syoubols to represent the normalized and unDormalized quantities. The coefficients $A(p)$ and $B(p)$ are given by Eqs. (6), suilably normalited. The integral term may be evaluated by replacing the indefinite limits in Eq. (7) by oo, giving when normalized

$$
r_{\left(\mathrm{x}_{3}\right) \approx \theta^{\mathrm{g} / \mathrm{a}}}\left(\frac{H_{a}(\theta, Z)}{v p}+\frac{H_{\phi}(\Theta, Z)}{t^{2}}\right)
$$

where $H_{a}$ and $H_{b}$ arc definite integrals of $\chi_{1}$ (and thus independent of momentum) that must be determined numerically. In the binit $\theta \rightarrow 0$, both $H_{a}$ add $H_{b}$ are faite. In normalized form with $p^{2} \gg \theta, F q$. (1T) reads

$$
\begin{aligned}
\frac{\theta V_{1}^{3}}{v^{3}}\left[\frac{\partial^{2} X_{1}}{\partial p^{2}}-\right. & \left.\left(\frac{v}{\theta}+\frac{3}{v \gamma^{3}}-\frac{2}{p}\right) \frac{\partial X_{1}}{\partial p}\right] . \\
& -\frac{1}{v p^{2}}\left(1+Z-\frac{\theta V_{1}^{2}}{v^{2}}\right) X_{1}+\theta^{3 / 2}\left(\frac{H_{x}}{v p}+\frac{H_{B}}{v^{2}}\right)+v=0 .
\end{aligned}
$$


The error in this equation is exponentially small.

We now make a subsidiary expanbion in small $\theta$. In the limit $\theta \rightarrow 0$, aeveral terms in Eq. (23) drop out leaving

$$
-\frac{1}{v^{2}} \frac{\partial \chi_{1}}{\partial p}-\frac{1+Z}{v p^{2}} \chi_{1}+v=0 .
$$

'fhis may by solved with the boundary condition $\chi_{1}(p=0)=0$ to give

$$
x_{1}=\left(\frac{\gamma+1}{\gamma-1}\right)^{\frac{1+\pi}{2}} \int_{0}^{z}\left(\frac{\gamma^{\prime}-1}{\gamma^{\prime}+1}\right)^{\frac{1+z}{2}} v^{\prime 3} d p^{\prime}
$$

This is the result derived using the Langevin equations by Fisch. ${ }^{2}$ For integer values of $Z$, the integral may be expresse. $J$ in terms of elementary functions. In particular for $Z=1$ we have

$$
x_{1}=\left(\frac{\gamma+1}{\gamma-1}\right)(v p-2 \log \gamma) .
$$

Of particular intereat is the efficiency for large $p$ since this gives a limit to the efficiency of current drive by fest waves. If we let $p>1$, the integral may be approximately evaluated to give

$$
x_{1} \rightarrow p-(1+Z) \log p \text {. }
$$

If we now take $\theta$ to be finite, Eq. (23) cannot be easily solved. However, we may solve it in the limit $p>1$. We echieve this by writing

$$
x_{1} \approx \alpha p+\beta \log p
$$

ia analogy to the situation with $\theta=0$. Subatituting this form of $\chi_{1}$ into $E q$. (23) and balarcing termo of equal order in $p$ gives

$$
\alpha=\frac{1+\theta^{9 / 2} H_{b}}{V_{t}^{2}}
$$

from the $O\left(p^{0}\right)$ terms and

$$
\beta=-\frac{\left(1+Z-3 \theta V_{t}^{2}\right) a-\theta^{3 / 2} H_{a}}{V_{l}^{2}}
$$

from the $O\left(p^{-1}\right)$ terms. When the if excitation is localized, the current-drive efficiency is given by Eqg. (18) that, with $x_{1}$ given by Eq. (25), read

$$
\begin{aligned}
& \frac{J}{P} \approx \alpha+\frac{\beta}{P} \\
& \frac{J}{P} \approx \beta \frac{1-\log p}{p}
\end{aligned}
$$


for current drive by Landau-damped and cyclotron-damped waves, respectively. |The factor of $1 / v$ in Eq3. (18) is roplaced by unity in the limit $p \rightarrow \infty$.] Equation (27a) (with p replaced by po) also applies for current drive by a narrow spectrum es given by $\mathrm{Eq}$. (19). In the limit of $p \rightarrow \infty$, the efficiency of cyclatronde rped current drive vanishes, while for current drive by Landau-damped waves $J / P \rightarrow \alpha$. In order to determine this limiting efficiency, either Eq. (26a) may be evaluated using the uumerically found value of $H_{0}(\theta, Z)$ (see Table II) or else the equation may be expanded as a series in $\theta$ to give for $p \rightarrow \infty$

$$
\frac{J}{P} \approx 1+\frac{5}{2} \theta+H_{b}(0, Z) \theta^{\mathrm{g} / 2} .
$$

$\mathrm{H}_{3}(0, Z)$ is tabulated in Table III.

We now turn to the salution of Eq. (17) in the nourelativistic limit $\theta \rightarrow 0$. We shall still consider only the limit $p>p_{i}$ The limits here are nonunilorm. Enquation (23) was obtrined by taking $p \gg p_{t}$ followed by $\Theta \rightarrow 0$ Here we will lake the lim :,$s$ in the opposite order. To do this, it is convenient to renormalize Eq. (17) using $q, m, p_{t}$, and $\nu_{t}$ as the nystem of units. In this case, $J / P$ is normalized to $q / p_{t} \nu_{t}$. Making this change of normalization and taking the limit $\theta \rightarrow 0$ is equivalent to formally replacing $\boldsymbol{\theta}$ by unity and substituting $v=\boldsymbol{p}$, $\gamma=1$, and $V_{t}^{2}=1$ in Eq. (23) to give

$$
\frac{1}{p^{3}}\left[\frac{\partial^{2} \chi_{1}}{\partial p^{2}}-\left(p+\frac{1}{p}\right) \frac{\partial \chi_{1}}{\partial p}\right]-\frac{1}{p^{9}}\left(1+Z-\frac{1}{p^{2}}\right) x_{1}+\frac{H}{p^{2}}+p=0
$$

where $H(Z)=H_{a}(0, Z)+H_{b}(0, Z)$ (this is tabulated in Table III). For $p>1$ (in this normalization this means $p \rightarrow p_{t}$ ), we may develop an asymptotic expression for $\chi_{1}$ as a series in powers of $p$. Balancing the terms in Eq. (29) from $O(p)$ (the leading order) to $O\left(p^{-4}\right)$ gives

$$
x_{\lrcorner}=\frac{p^{4}}{5+Z}+\frac{9 p^{2}}{(5+Z)(3+Z)}+\frac{H p}{2+Z}+\frac{9}{(5+Z)(3+Z)(1+Z)}+O\left(p^{-2}\right) .
$$

For localized excitation, Eq. (18) becomes

$$
\begin{aligned}
& \frac{J}{P}=\frac{4 p^{2}}{5+Z}+\frac{18}{(5+Z)(3+Z)}+\frac{H p^{-1}}{2+Z}+O\left(p^{-4}\right) \\
& \frac{J}{P}=\frac{3 p^{2}}{5+Z}+\frac{9}{(5+Z)(3+Z)}-\frac{9 p^{-2}}{(5+Z)(3+Z)(1+Z)}+O\left(p^{-1}\right)
\end{aligned}
$$

for Landau-damped waves and cyclotron-damped waves, respectively. The leading order terms here (those proportional to $p^{2}$ ) are exactly those derived by Fisch and Bowser. ${ }^{9}$

In order to conpute the efficiencien for current drive by a narrow spectrum of waves, it is neceggary to carry out the integrations in Eqg. (19) and (20). The 
following ayymptotic series is useful for this purpose:

$$
\int_{x}^{\infty} \exp \left(-\frac{1}{3} y^{2}\right) y^{n+1} d y=\exp \left(-\frac{1}{2} x^{2}\right)\left(x^{n}+n x^{n-2}+n(n-2) x^{n-1}+\cdots \cdot\right] \text {. }
$$

For $n$ even, the seriea terminated and is exact. The efficiency for current drive by a narrow spectrum of Landau-damped waves, Eq. (19) becomes

$$
\frac{J}{P}=\frac{4 v_{p}^{2}}{5+Z}+\frac{6(6+Z)}{(5+Z)(3+Z)}+\frac{H v_{p}^{-1}}{2+Z}+O\left(v_{p}^{-2}\right) .
$$

For a nartow apectrum of cyclotron-demped wavea, Eq. (20) gives

$$
\begin{aligned}
& \frac{J}{P}=\frac{3 v_{p}^{2}}{5+Z}+\frac{3(9+2 Z)}{(5+Z)(3+Z)}+O\left(v_{p}^{-2}\right), \\
& \frac{J}{P}=\frac{3 v_{p}^{2}}{5+Z}+\frac{9(1+Z)}{(5+Z)(3+Z)}+O\left(v_{p}^{-2}\right),
\end{aligned}
$$

for $l=1$ and $l=2$, reapactively. The effect of the integrations is to change only the higher-order $O\left(v_{p}^{0}\right)$ corrections to the efficiencies. The leading order termg are the same an for tine localized excitation Fq3. (30). Equations (31) and (32a) are plotted in Fig. 4 . These closely approximate the exact resulte for $v_{p}>2 v_{t}$

\section{HIGH ENERGY LIMIT OF COLLISION OPER- ATOR}

In the previous section, we derived figite temperature corrections to the efficiency limit found in Rel. 2. However, the collibion operator in the Landav form Eqa. (1) was derived by apeuming that the background electrons are only weakly relativiatic or that $\theta<1$. We must check, therefore, that the finite $\theta$ corrections to the Landau operator do not sfect the formula for the efficiency limit Eq. (2B).

The lineariatd collision operator Eq. (10) conists of three collision terms. Since in all practical cases the ions are nonrelativistic, the ion term $C\left(f, f_{i}\right)$ needs no correction. The term $C\left(f_{\mathrm{m}}, f\right)$ contributes to the integral term $I\left(\mathrm{x}_{1}\right)$ in Eq. (17). However, thia resulted in a $O\left(\theta^{\mathrm{s} / \mathrm{a}}\right.$ ) cont ribution to efficiency limit Eq. (28), ao that corrections to this term will be of bill higher order.

Therefore, we need only congider collisions off a Maxwellian electron background $C\left(f_{1}, f_{m}\right)$. Furthermore, if $\theta$ is small and if $p>p_{1}$, we may take $v^{\prime} \leftarrow v$ in the full collision kernel $E_{9}$. (2) and approximate $U$ by its Taylor expansion about $v^{\prime}=0$. By retaining terms up to second onder in $v^{\prime}$, we obtain

$$
C\left(f, f_{m}\right)=\frac{\Gamma}{2} \frac{\partial}{\partial p_{j}}\left(U_{j k}^{(0)} \frac{\partial f}{\partial p_{k}}+\frac{\partial U_{j k}^{(0)}}{\partial v_{k}^{\prime}} \frac{v_{t}^{2}}{T} f+\frac{1}{2} \frac{\partial^{2} U_{j k}^{(0)}}{\partial v_{m}^{\prime} \partial v_{m}^{\prime}} v_{i}^{2} \frac{\partial f}{\partial y_{k}}\right)
$$


where summation over repeated indices is implied and the superscript $(0)$ is used to indicate that $U$ and its derivatives are evaluated at $\mathbf{v}=0$. Evaluating these coefficients gives

$$
\begin{aligned}
U_{j k}^{(0)} & =\frac{v^{2} b_{j k}-v_{j} v_{k}}{v^{3}}, \\
\frac{\partial U_{j k}^{(0)}}{\partial v_{k}^{\prime}} & =\frac{2 v_{j}}{v^{s}}, \\
\frac{1}{2} \frac{\partial^{2} U_{j k}^{(0)}}{\partial v_{m}^{\prime} \partial v_{m}^{\prime}} & =\frac{2 v_{j} v_{k}}{v^{\delta}}-\left(1-\beta^{4}\right) \frac{U_{j k}^{(0)}}{v^{2}} .
\end{aligned}
$$

(This calculation was carried out using MACSYMA. ${ }^{22}$ ) If we compare these witt the equivalent expreasjona using $U$ from Eq. (1c), we find that only the term proportional to $\beta^{4}$ is new. The high energy form of $C\left(f, f_{m}\right)$ is given by Eq. (4a) with $A(p)$ given by Eq. (6a), $F(p)=(v / T) A(p)$, and

$$
B(p)=\Gamma \frac{1}{2 v}\left[1-\frac{v_{t}^{2}}{v^{2}}\left(1-\frac{v^{4}}{c^{4}}\right)\right] .
$$

In other words, in the high-energy limit the electromagnetic correction only changes the pitch-angle scattering term. The new term hag no effect on the asymptotic form for the efficienciea Eq. (27) because it is am: vler by $\beta^{t}$ than another term in $B$, which had no effect.

Connor and Hastie ${ }^{21}$ also give an expression for collisions of bigh-energy particles aff a fixed background. The corrections to Eq. (6) that they obtain differ from ours. This is posaibly because the background distribution that they treat is only approximately Marwellian.

\section{CONCLUSIONS}

We have conaidered the problem of current drive by fast waves in a relativistic plasma. Let ua briefly review the approximations made. The major one is the reduction of the full colligion operator to Landau form. We show in Sec. II that this holds if the background temperature is small, $T<\mathrm{mc}^{2}$. The corrections to the Landau operator in the high energy limit are derived in Sec. VII and are shown to be amall. The subsequent analygis leading to the formula for the current-drive efficiency Eq. (15) is exact. In order to apply this formula, it is necessary to determine the rf-induced fux $S$ from Eq. (8) and the Spitzer-Härm function $x$ from Eq. (13).

We considered two methods for computing S: either to assume that $f=f_{\mathrm{m}}$ in Eq. (8) (corresponding to linear damping) or to solve the two-dimensional Fokker-Planck equation, Eq. (22), numerically. The latter method may be necessary in the case of high $r$ powers and wide spectra. Nate that the efficiency 
can be accurately calculated even if the $\mathbf{S}$ is known only approximately aince Eq. (15), being an integral operator on $\mathbf{S}$, is insensitive to amall errora in $\mathbf{S}$. Ofter, ueful information can be extracted from Eq. (15) even with very limited information about $\mathbf{S}$. If the if epectrum is known, we can make some estimates (based on either numerical or epproximate analytical aolutions to the FokkerPlanck equation) of where in momentum apace the fux is largeat. We can then use Eq. (16) to give the efficiency.

The Spilzer-Härm function $\chi$ can be determined by solving Eq. (17) numerically as in Sec. V. Since this equation is just a coe-dimensional equation, there is little difficulty in obtajning arbitrarily accurate results in this way. This method can be regarded as exact. Aiternativriy, we found asymptotic forms for $\chi$ in Sec. VI. From this we can write down analytical expressions for the current-drive efficiency in various cages as given in Eqs. (27), (28), (30), (31), and (32).

The primary application of this work is, of couree, to maintain a steady-state toroidal current in a tokamak reactor. The viability of thie schene depends upon the amount of circulating power that is required. Thus, an accurate calculation of the current-drive efficiency, as well as an assessment of the best possible efficiency, are of crucial importance.

When applying these results to the study oi steady-state current drive in a tokamak, it is useful to convert the efficiency $J / P$ to $I / W$ where $I=A J$ is the total current, $W=2 \pi R A P$ is the total $n$ power, $A$ is efiective poloidal crass-bectional area, and $R$ is the tokamal major radius. This gives

$$
\begin{aligned}
\frac{I}{W W} & =\frac{1}{2 \pi R} \frac{J}{P} \\
& =2.08 \frac{J / P}{q / m c \nu_{c}} \frac{10^{20} \mathrm{~m}^{-3}}{n} \frac{1 \mathrm{~m}}{R} \frac{15}{\log \mathrm{A}} \mathrm{A} / \mathrm{W} \\
& =40.7 \times 10^{-3} \frac{J / P}{q / \rho_{t} \nu_{t}} \frac{10^{20} \mathrm{~m}^{-3}}{n} \frac{T}{10 \mathrm{keV}} \frac{1 \mathrm{~m}}{R} \frac{15}{\log \mathrm{A}} \mathrm{A} / \mathrm{W} .
\end{aligned}
$$

The last two equalitieg give the conversion from the normalized efficiencies given in the Ggures and in Sec. VI to practical units. Figures 2, 3, and 4 contain scales in these units.

The present work calculates the efficiency that can be expected from an arbitrary wave-induced flux. It is possible, therefore, to come to some very general conclusiona about the best posgible efficiency that can be obtained by driving currente with different waves. In particular, there is a limit, given by Eq. (28), to the efficiency of current drive with fast waves, auch as lower-hybrid waves, that interact through a Landau resonance with relativistic electrons. These waves are, perbaps, the most likely candidate for current drive in a reactor.

The present calculations also apply to other types of curtent drive, for example, relativistic electron beams 29 Here, the efficiencies will be similar to those of Landau-damped waves. Care must be taken, however, in interpreting 
experiments on relativistic electron beams because the assumption of a steady state is generally inapplicable.

The equationg developed here apply to other forme of if curreat drive. Sonne of these may be very efficient, more so than lower-bybrid wave-induced fluxes. For example, if low-phase-velocity waveg interact through a cycjotron resonance with fast electrons, the if flux may be nearly parallel to the constant energy contours, at the same time that the collisionality of the resonant electrons is small. This gives very high effic:ency, but, is practice, these waves are much more difficult to generate than are lower-hybrid waves.

Settling the question of the highest attainable current-drive eficiency with fast waves should enable, we hope, tokamak reactor designers to asseso the practicality of uging waves to drive steady-gtate currents. There may, of courge, be other effects that present difficulties, such as the accessibility of the waves $o_{i}$ nonlinear effecto. On the other hand, there may be effecte, such as the hootstrap current, which could be helpful.

Finally, we hope that the form that we derived bere for the reiativistic colligiou operator, which enabled us to solve for the relativistic Spitzer-Härm function, will be of use in other numerical problems dealing with collisions in hot plasmas.

\section{ACKNOWLEDGMENTS}

This work was gupported by the United States Department of Energy un: er Contract DE-AC02-76-CHO-3073. 


\section{REFERENALS}

${ }^{1}$ N. J. Figch, Phys. Rev. Lett. 41, 873 (1978).

${ }^{2}$ N. J. Fiech, Phys. Rev. 34A, 3245 (1981).

${ }^{5}$ T. M. Antonsen and K. R. Chu, Phys. Fluids 25, 1295 (1982).

D. J. H. Wort, Plasma Phya, 13, 258 (1971)

${ }^{5}$ C. F. F. Karney and N. J. Firch, Phys. Fluids 22, 1817 (1979).

${ }^{8}$ N. J. Fiach and C. F. F. Karney, Phys. Fluida 24, 27 (1881).

7R. W. Harvey, K. D. Marx, and M. G. MeCoy, Nucl. Fusion 21, 153 (1981).

${ }^{8}$ C. F. F. Karaey and N. J. Fisch, Nucl. Fusion 21, 1549 (1981).

9N. 3. Fioch and A. H. Booser, Phys. Rev. Lett. 46, 720 (1980).

10J. G. Cordey, T. Edlington, and D. F. H. Start, Plama Phyo. 24, 73 (1982).

IIM. Tałuchi, J. Phys. Soc. Jpn. 62, 2035 (1983).

17g. P. Hirshman, Phys. Fluida 23, 1238 (1980).

${ }^{19}$ M. Taguchi, J. Phys. Soc. Jpn. 51, 1975 (1982).

${ }^{14}$ L. Spitrer and R. Härm, Phys. Rev. 89, 977 (1953).

${ }^{15}$ K. Hizanidis and A. Bera, Plasma Fumion Center Report PEC/JA-84-4, Magsachusetts Inotitute of Technolog (1984) [oubmitted to Phys. Fluids].

16. T. Belinev and G. 1. Budker, Sov. Phys. Doklady 1, 218 (1958).

${ }^{17}$ L. D. Landau, Phys. Z. Sowjet. 10, 154 (193A).

${ }^{10} \mathrm{M}$. N. Rosenbluth, W. M. MacDonald, and D. L. Judd, Phy. Rev. 107, 1 (1957).

IPS. R. de Groot, W. A. van Leeuwen, and Ch. G. van Weert, Relativisic Kinetic Theory (North-Holland, Amoterdam, 1980).

${ }^{20} D$. Mosher, Phys. Fluids 18, 846 (1975).

21 J. W. Connor and R. J. Hastie, Nucl. Fusion 15, 415 (1975).

${ }^{22}$ The Mathlab Group, MACSYMA Refenence Manual, Vervion 10, Laboratory for Computer Science, Mansacbusetts Inatitute of Technology (1983).

${ }^{29}$ M. G. McCoy, A. A. Mirin, and J. Kileen, Computer Phyo. Comm. 24, 37 (1981).

94. Chapman and T. G. Cowling, The Mathematical Theory of Non-uniform Gages, 3rd edition (Cambridge Univeraity Prese, Cambridge, 1970).

${ }^{25}$ C. F. Kennel and F. Engelmann, Phys. Fluids 9, 2377 (1986).

${ }^{20}$ S. I. Braginakiu, in Reviews and Plasma Physics, Vol. 1 (Conaultanto Bureau, New York, 1965), p. 205.

${ }^{\text {} 7}$ J. G. Cordey, E. M. Jones, D. F. H. Start, A. R. Curtis, and I. P. Jones, Nucl. Fusion 10, 249 (1979).

${ }^{28}$ R. A. Gairns, J. Owen, and C. N. Lashmore-Davies, Phys. Fluids 28, 3475 (1983).

${ }^{20}$ V. L. Bailey, J. M. Creedou, B. M. Ecker, and H. I. Helav, J. Appl. Phys. 54, 1656 (1983). 


\section{TABLES}

TABL.E $I$. The coefficients for the efficiercy for the three types of current drive by, low frequency waves.

\begin{tabular}{cccc}
\hline$Z$ & $\overline{C_{L}}$ & $\overline{C_{H}}$ & $\overline{C_{A}}$ \\
\hline 1 & 3.76 & 8.19 & 8.09 \\
2 & 1.88 & 5.17 & 5.07 \\
5 & 0.75 & 2.55 & 2.60 \\
10 & 0.38 & 1.42 & 1.48 \\
\hline
\end{tabular}

TABLE il. Table of efficiencies $J / P$ for Landas-danped waves in the limit $v_{p} \rightarrow c$. The efficiencies are normalized to $q / m c \nu_{c}$.

\begin{tabular}{ccccc}
\hline $\bar{\theta}$ & $\bar{Z}=1$ & $\bar{Z}=2$ & $\bar{Z}=5$ & $\bar{Z}=10$ \\
\hline 0.01 & 1.04 & 1.03 & 1.03 & 1.03 \\
0.02 & 1.09 & 1.07 & 1.06 & 1.06 \\
0.05 & 1.25 & 1.20 & 1.17 & 1.15 \\
0.1 & 1.55 & 1.44 & 1.34 & 1.30 \\
0.2 & 2.19 & 1.91 & 1.70 & 1.81 \\
\hline
\end{tabular}

TABLE III. The coefficients $H_{\mathrm{a}}(0, Z)$ and $H(Z)$.

\begin{tabular}{crr}
\hline$Z$ & \multicolumn{1}{c}{$H_{a}$} & \multicolumn{1}{c}{$H$} \\
\hline 1 & 13.69 & 21.12 \\
2 & 9.13 & 13.51 \\
5 & 4.94 & 7.01 \\
10 & 2.88 & 4.01 \\
\hline
\end{tabular}




\section{FIGURES}

FIG. 1. Contour plots of $x(p)$ for $Z=1$ and (a) $\theta=0$ and (b) $\theta=0.01$. The contour levels are evenly spaced with increments of $50 q p_{s} / m \nu_{t}$. The higher levelo are on the right (i.e., $\partial \chi(p) / \partial p_{\beta}>0$ ).

FIG. 2. EGiciencies for localized excitation for (a) Landau-damped waves (parallel diffusion) Eq. (18a) and (b) cyclotron-damped waves (perpendicular diffusion) Eq. (18b). The different mrves show the efficiencies for various valuea of the temperature $\theta$ as indicated. In ail cases $Z-1$. The top scale give: the kinetic energy of the electrons. The right acale gives the efficiency for a plasme with $n=10^{20} \mathrm{~m}^{-3}, \log \Lambda=15$, and $R=1 \mathrm{~m}$.

FIG. 3. Efficiencies for narrow Landau gpectrum Eq. (19) as a function of the phasc "slocity $v_{p}$. The curves correspond to the various values of $\theta$. In all cases $Z=1$. The top acale gives the paralle! index of refraction $n_{\|}=c / v_{p}$. The right acale gives the efficiency for the same conditions as in Fig. 2.

FIG. 4. Efficiencies for narrow spectra of Landau-damped (L) waves and cyclotron-damped $(C)$ waves $(l=1)$ for the nonrelativintic case $\theta \rightarrow 0$ and $Z=$ 1. Also shown as jashed lines are the asymptotic results $:-3$. (31) and (32a). The right acale gives the efficiency for a plasms with $n=10^{20} \mathrm{~m}^{-9}, T=$ $10 \mathrm{keV}, \log A=15$, and $R=1 \mathrm{~m}$.

FIG. 5. Contour plot of the steady-state distribution $f$ obtained by numerically integrating Eg. (22). Here $Z=1, \theta=0.01, v_{1}=0.4 c_{1} v_{2}=0.7 c$. The resonant region is indicated. 'The contour levels are chosen oo that for a Maxwellian they would be equally spaced with $\Delta p=m c / 30$. 

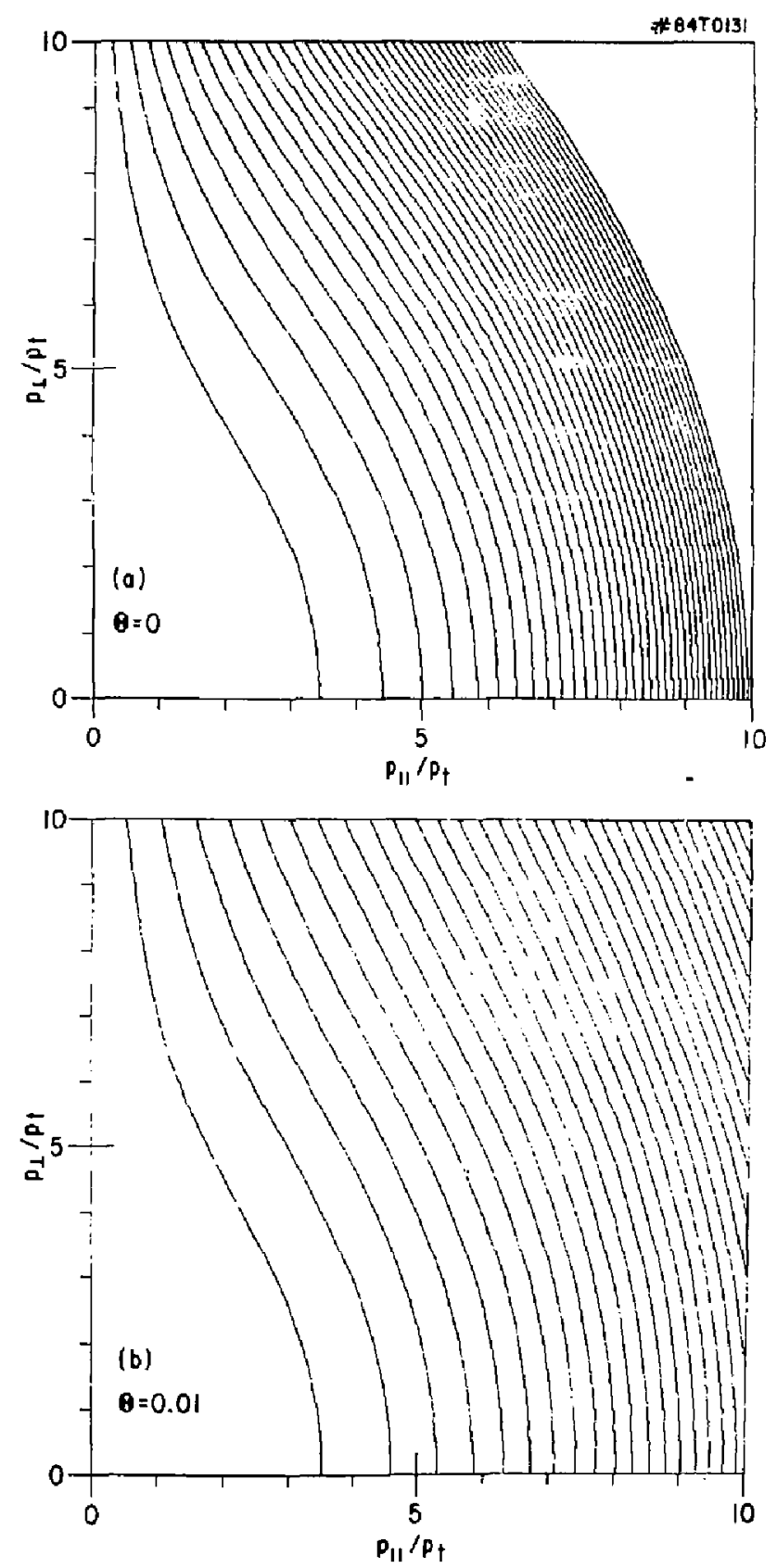

Fiq. 1 

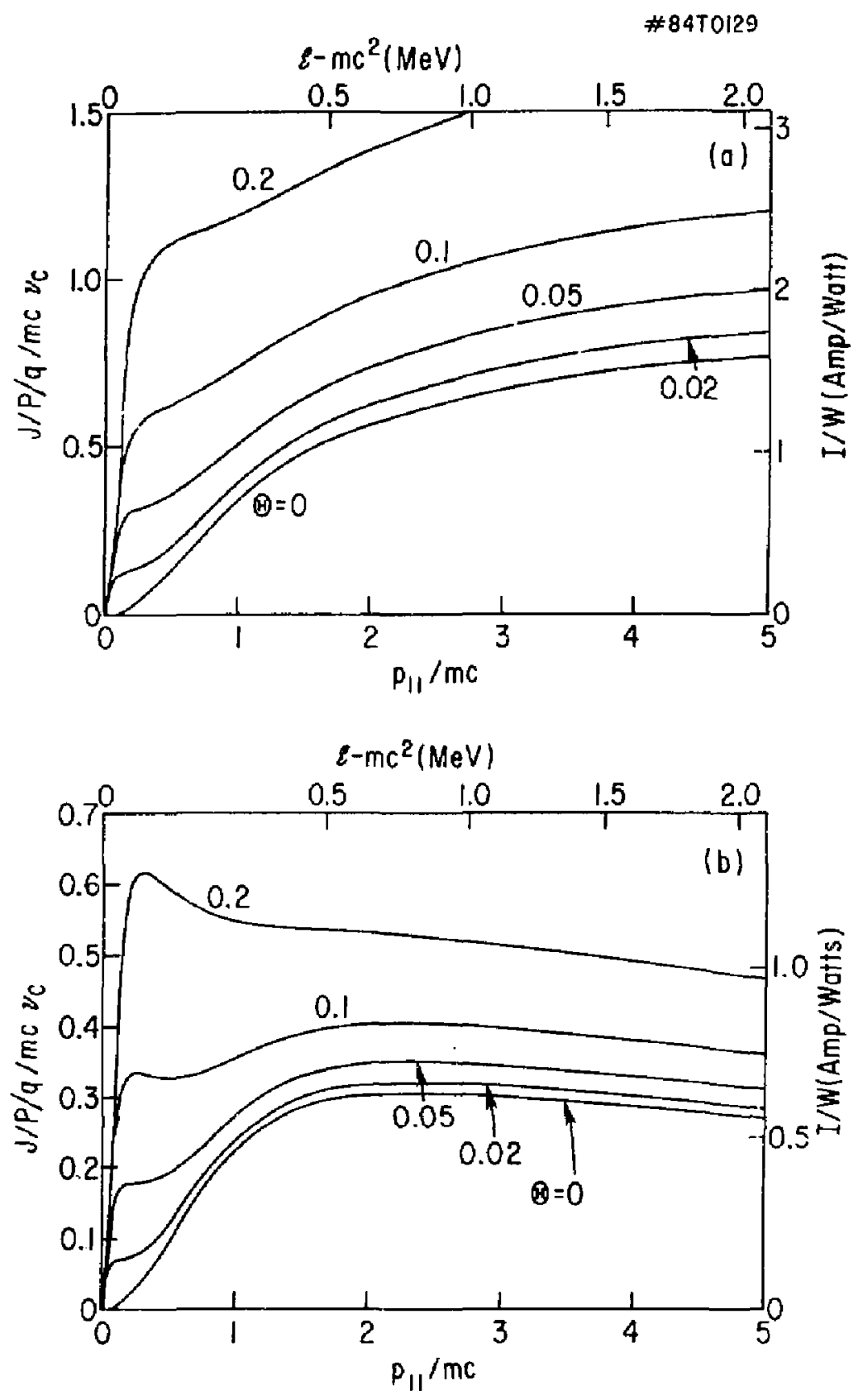

Fig. 2 


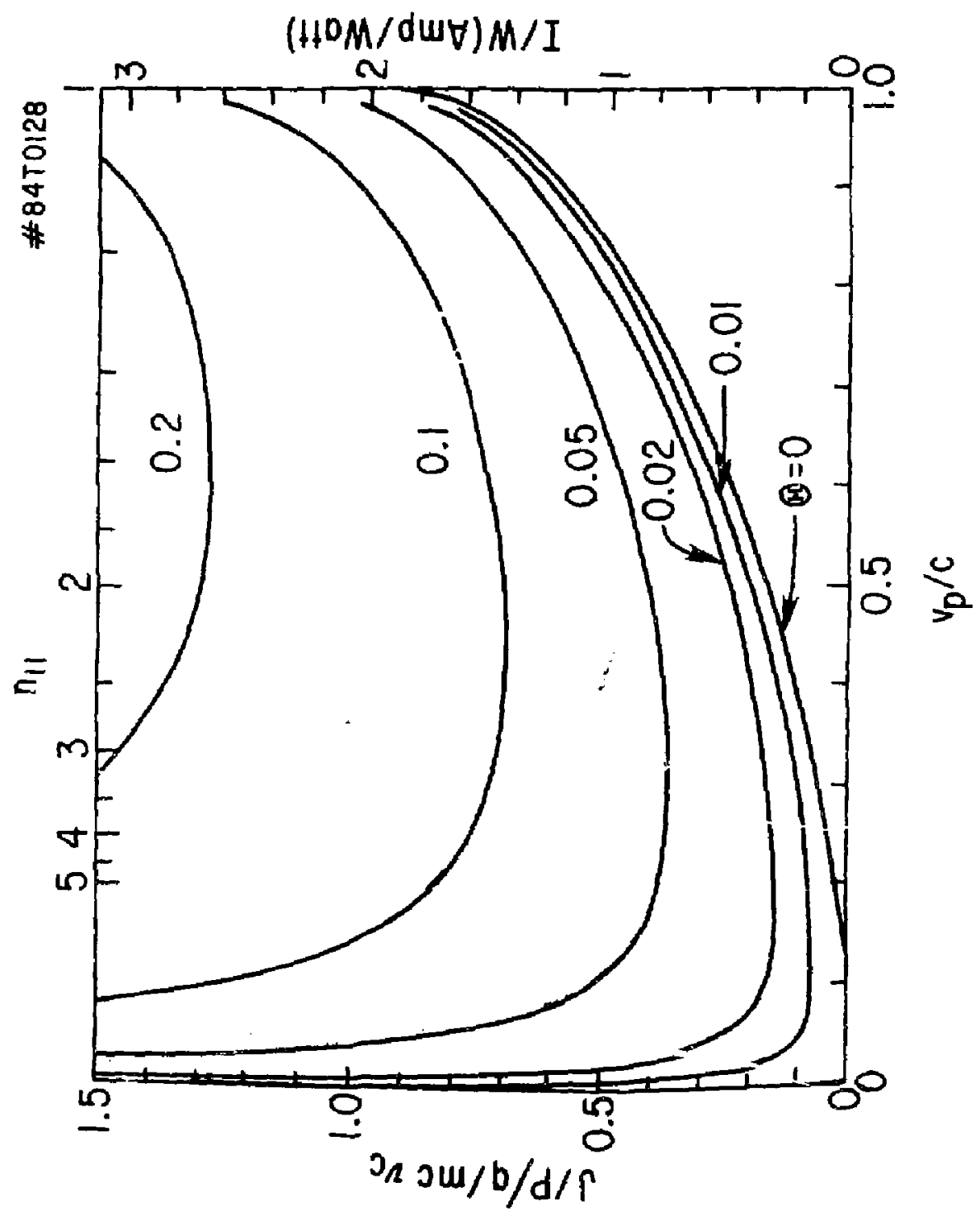

$\underset{3}{m}$ 


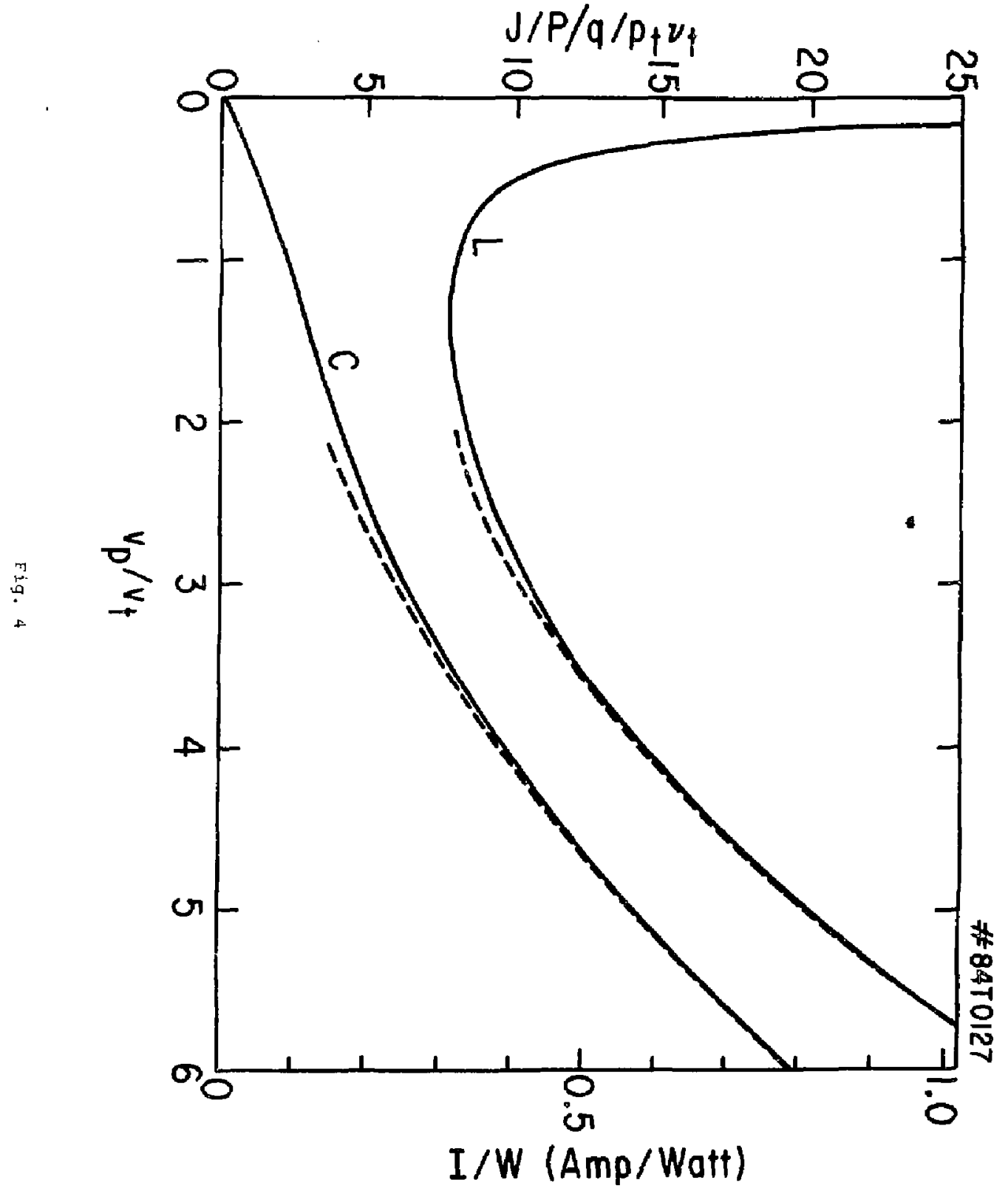




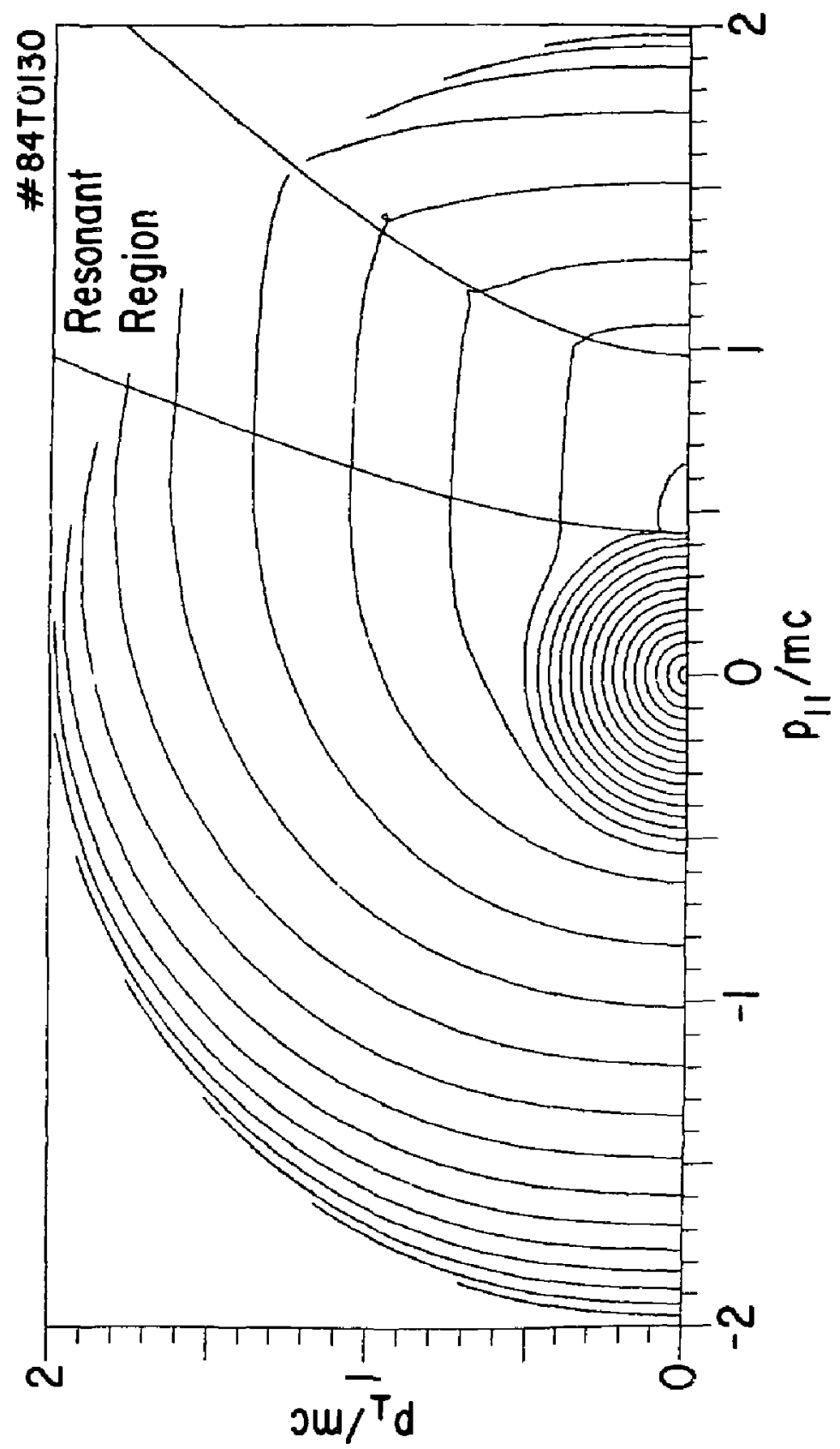

ज 
Plosm fies Lob, Austro Not'I UnIQ, AUSTRALIA Dr. Fronk J. Pootonl, Urily of wollongang. AuStRal ta Prof. I,R. Jones, Filinders Uniz., Austral ia Prof. M.H. Erennan, Unio Syanoy, AUSTRML IA Prot. F. Cop, Inst Theo Pays, MUSikik Prol. Frank Vechoest, Inst theoretiseha, BelgILM Dr. D. Polumbo, Dg Xil Fusion Prog, Belgium Ecole Royale milltolre. Lob of Phrs Plasmos, BELGIJM Dr. P.H. Sakenoka, Unir EstQOnel, GRAZIL Dr. C.R. Jomes, Univ of Albetts, CANADA Prot. J. Teichmonn, Univ of montrost, CANADA Dr. H,H, Skarsgara, Unly of Soskatchomen, CANAOA Prot, S.R. Sreanivasan, Univarsity ol Colgery, CANADR Prot. Tudar hi. Jonnston, INRS-Energle, CANADA Dr. Hannes Bornard, Unle B-lyisn Columbla, CANADA Dr. M.F. Boehynski, ME Technologios, Hnc., CANADA Zhongmu L1, SW Ins: Physics, CHINA

Library. Is ing Hua University, Chila Llorarian, Institute of Physlcs, CHINA Inst Plesme Phys, Acodenle Sinlca. CHima Dr. Peter Lukge, Komenskeho Univ, CzECHOSLOVAKIA The Librat len, Cuingm loboratory. ENGLANo Prot. Senetzman, Deservatoire oe hice, FRance J. Rodet, CEHEPG, FRAnEE

AM Dufas Library, AM Dupas Llorary, FRANCE Dr. Tom Mual, Acadersy BI Dliegrephlc, MONG KONG Preprint Llarary. Cent Res Inst. Phys, HuNGapr Pr. 5.K. Irohan, Ponjov University, INOik Dr. Inora, Mohen lel Das, Banaras Hindu UnIv, INDIA Dr. L.K. Chavda, South Gujaret UnIr, INDiA Dr. R.K. Chnojlani, Ver Ruenl Morg. InDIA P. Kam. Pnýsical Resec ch Lat. InDIA Dr. Phlllid Rocmau, Isroel Inst Tech, ISRAEL Prof. S. Cupermon. Tel Aviv Universlty, ISRAEL Prot. G. Rostagni, Univ Di Podova, ITAly Llorarian, Int'j Ctr Tneo Pnys, Italy MISS CIGI!S DE Palo, Assoc ELRATOM-CNEN, ITALY Elbliotece, del CNA EURATON, ITALY

Dr. H. Tanato, Tosnlbe Fes \& Dev, JaPan Prot. N. Yoshlmano, JaERl, Toxoi Res ist, Japan Prot. UChide, UnI versity of iohyo, JAPAN Rese: rr Info Cerifer. Nogoro Unlversity, JaPAs Fro. Kyoji Nishlkowo. Univ of KIroshlme, JAPAk Prel. Sigeru Norl, JaERt, JaDah

LIbrory, Kyoto UniversIIy. JAPAN

Piot. Ichlre kbuakbmi, Nilhori Unit, JAPAN

Prof. Setosril Iton, Kyustitu Unluarsity, JaPAN

Teen Into Dlyisian, Kor ae ATomla Energy, KOREA Dr. R. Englend, Civiod IJnlversitorla, ilExICO Blollotheok, Fommlnst vor Plasmb, NETHERLAHDS Prot. E.5. Llley, Unlwerslay do walkato, NEw ZEALAND Dr. Suresh C. Sherma, Univ of Colober, NIGERIA
Prot. J.A.C. Codral, Inst Suder jor Tech, PORTueal Dr. Octevlon Petrus, ALI CLEA Unimorsity, Romania

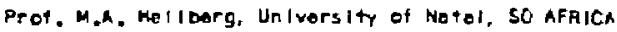
Dr. Johan de VIIlliers. Atom! Enorgy BO, SO AFRiCA Pusion Div, LIbrary, JEH, SPAJi

Prot. Hans Wilinelmsan, Chalners Univ Teen, SwEDEN Dr. Lennart Stent 10, Unlverslty of UMEA, SWEDEN Llarory, Royol Insi TeEn, SWEDEN

Dr. Erlk T. Kerison, Uppselo Untversitet, SWEDEN Centra de Rocherchesan, Ecole Polytoch Fod, SWITZERLand Dr. W.L. Welse, Npt'l Bur Stand, USA

Dr. W,M. Stacey, Georg Inst lech, USA Dr. 5.T. Wu, Un Iv Alaboms, USA

Frot. Normon L. Dlosan, Un it 5 Fiorldo, USA Dr, Benjamin Ma, lowe Stato Univ, USA Prol. Megne Kristlensen, Toxas Tecn Univ, USA Dr. Reymond Aske-, Auburn Unly, USA Dr. V.T. Tolok, Kharkav Phys Tech Ins, USSE Dr. D.D. Ryutoy, SI borlan Acad ScE, US5F Dr. G.A. Ellsoer, Kurenator Institute, USSF Dr. V.A. Gluknikh, Inst Electrof nysleol, USSF Institute Gen. Physles, US5R

Prof. T.J. Boyo, Univ Colloge n Holes, WALES Dr. K. Sehlnalor, Runr UnIversitot,, G G Nuc loar Res Estab, Juliori LtG, T. GERomit LIbrarlem. Maxtlanck. Instltut, M. GERANT

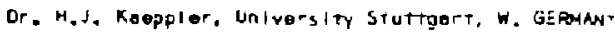
Blollothak, Inst Plasmelor schung, in. Geraman: 